\title{
Universiteit
}

Leiden

The Netherlands

\section{Polarization entanglement in a crystal with threefold symmetry}

Visser, J.; Eliel, E.R.; Nienhuis, G.

\section{Citation}

Visser, J., Eliel, E. R., \& Nienhuis, G. (2002). Polarization entanglement in a crystal with threefold symmetry. Physical Review A, 66, 033814. doi:10.1103/PhysRevA.66.033814

Version: $\quad$ Not Applicable (or Unknown)

License: $\quad$ Leiden University Non-exclusive license

Downloaded from: https://hdl.handle.net/1887/61330

Note: To cite this publication please use the final published version (if applicable). 


\title{
Polarization entanglement in a crystal with threefold symmetry
}

\author{
J. Visser, ${ }^{*}$ E. R. Eliel, and G. Nienhuis \\ Huygens Laboratorium, Universiteit Leiden, Postbus 9504, 2300 RA Leiden, The Netherlands
}

(Received 12 June 2002; published 20 September 2002)

\begin{abstract}
Polarization entanglement of twin photons created in the process of parametric down-conversion is fully determined by the pump polarization when the pump, signal, and idler beams are collinear with the symmetry axis of the nonlinear crystal. We point out that in this situation a threefold rotational symmetry is needed for the process to occur. We describe the polarization entanglement of the twin photons in terms of correlations on the Poincare sphere. The inherent nonconservation of the intrinsic angular momentum of light in this process is discussed.
\end{abstract}

DOI: 10.1103/PhysRevA.66.033814

PACS number(s): 42.65.An, 42.50.Ct, 03.65.Ud

\section{INTRODUCTION}

Polarization entanglement between pairs of photons is commonly created in the process of spontaneous parametric down-conversion (SPDC), where one photon absorbed from a pump beam in a nonlinear crystal leads to the creation of two photons, which are called signal and idler photons. The crystal must have a nonvanishing second-order nonlinear susceptibility, and polarization-entangled pairs are selected by appropriate filtering of the down-converted light.

In general, the orbital and intrinsic angular momenta of the light field are not conserved in the SPDC process [1,2]. In the special case that pump, signal, and idler beams have a common axis, the orbital angular momentum along this axis is conserved at the single-photon level [3-5]. This follows basically from the overlap integral of the pump field and the product of signal and idler, which involves the integral $\int d \phi \exp \left[i \phi\left(l_{p}-l_{s}-l_{i}\right)\right]$, with $\phi$ the azimuthal angle in the transverse plane, and $l$ the azimuthal mode index, which determines the orbital angular momentum $l \hbar$ per photon [6-9].

In the present paper we analyze the polarization entanglement of twin photons and the corresponding intrinsic angular momentum in a spherical basis using the Poincaré sphere. As an example we discuss the case of a crystal with $C_{3 v}$ symmetry, where the propagation directions are chosen to coincide with the symmetry axis of the crystal. As pointed out by Bloembergen [10] in the context of second-harmonic generation, for this case a circularly polarized fundamental mode is converted into a harmonic with the opposite circular polarization. In a similar fashion, during SPDC, a circularly polarized pump photon creates a signal-idler photon pair with the opposite circular polarization. For a pump photon with an arbitrary polarization, a polarization entangled photon pair is created. Obviously, the intrinsic angular momentum is in general not conserved during this process. The change in angular momentum of the light field is compensated by that of the crystal medium.

In Sec. II we discuss a representation of the two-photon polarization state on the Poincare sphere and we consider the intrinsic angular momentum associated with the polarization. We shall show that, for the process of SPDC, the polarization

*URL: http://molphys.leidenuniv.nl/qo/index.html entanglement of the photon pair is determined by the polarization of the pump and by the nonlinear susceptibility of the crystal. In Sec. III we derive properties of the susceptibility tensor $\chi^{(2)}$ from symmetry arguments. We do this in the basis of spherical unit vectors, which is unusual, but powerful and elegant. In Sec. IV we use the results from previous sections to study the polarization entanglement and the associated intrinsic angular momentum of twins created in a crystal with $C_{3 v}$ symmetry. We end with conclusions in Sec. V.

\section{REPRESENTATION OF THE TWO-PHOTON POLARIZATION STATE ON THE POINCARÉ SPHERE}

\section{A. Single-photon polarization states}

The polarization vector of a light beam with a given propagation direction can always be expressed as a linear combination of the circular polarization vectors $\vec{u}_{ \pm 1}$. We take the propagation direction parallel to the $z$ axis, in which case the circular polarization vectors are given by

$$
\begin{gathered}
\vec{u}_{+1}=-\frac{1}{\sqrt{2}}(\vec{x}+i \vec{y}), \\
\vec{u}_{-1}=\frac{1}{\sqrt{2}}(\vec{x}-i \vec{y}),
\end{gathered}
$$

where $\vec{x}$ and $\vec{y}$ are Cartesian unit vectors. For one photon, these polarization states are denoted as $| \pm\rangle$. When the states $|+\rangle$ and $|-\rangle$ are mapped onto the states up and down of a spin $1 / 2$, each polarization state is equivalent to a specific spin state. A pure state of a spin $1 / 2$ is uniquely determined by the expectation value $\langle\vec{S}\rangle$ of the spin vector, which always has a length of $1 / 2$. Therefore, such a state can be represented in a unique way as a point on a sphere, commonly called the Bloch sphere. Because of the aforementioned mapping, a pure polarization state is also represented by a point on a sphere; the latter is named after Poincaré. The spherical coordinates $\theta$ and $\phi$ of this point fully determine the polarization state. The poles of the Poincare sphere correspond to opposite circular polarizations, and points on the equator represent states of linear polarization. The ellipticity of the polarization is determined by the polar angle $\theta$, such that the 


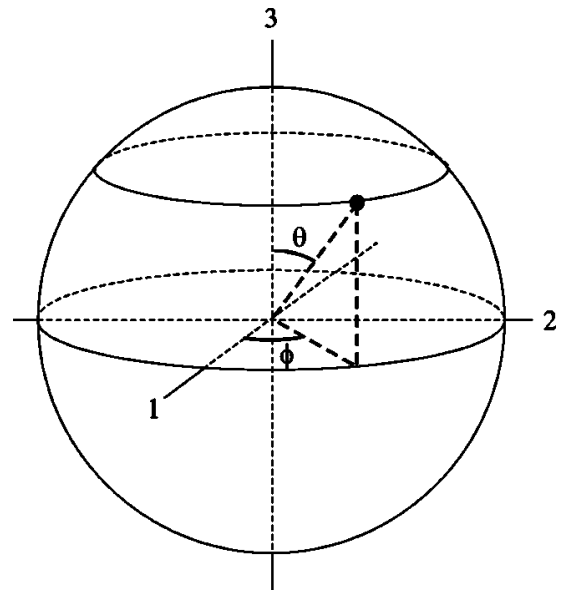

FIG. 1. Representation of the one-photon polarization state on the Poincaré sphere. If $\theta=\pi / 2$, we have linear polarization in the $y$ direction for $\phi=0$ and in the $x$ direction for $\phi=\pi$. For $\theta=0$ and $\theta=\pi$, we have right- and left-handed circular polarizations, respectively.

cross product $\vec{e} \times(\vec{e})^{*}$ of the polarization vector and its complex conjugate has the strength $\cos \theta$. The angle between the long axis of the ellipse and the $x$ axis is equal to $(\pi$ $+\phi) / 2$, where $\phi$ is the azimuthal angle. The absolute value of the overlap $\left|\vec{e}_{1}^{*} \cdot \vec{e}_{2}\right|$ of two polarization states is given by $\cos (\alpha / 2)$, where $\alpha$ is the angular distance between the corresponding points on the Poincare sphere. Consequently, opposite points on the Poincare sphere always correspond to orthogonal polarizations. The polarization of a photon that corresponds to the point on the Poincare sphere with polar angle $\theta$ and azimuthal angle $\phi$ is then given by (see Fig. 1)

$|\theta, \phi\rangle=\cos (\theta / 2) \exp (-i \phi / 2)|+\rangle+\sin (\theta / 2) \exp (+i \phi / 2)|-\rangle$.

\section{B. Two-photon polarization states}

Now we use the Poincare representation for the description of the entangled polarization state $|\Psi\rangle\rangle$ of twin photons, where the double ket is used to indicate that it is a twophoton state. When one photon is detected in a selected polarization state $\left|\psi^{(1)}\right\rangle=\left|\theta_{1}, \phi_{1}\right\rangle$, the state of the remaining photon collapses into the state $\left|\psi^{(2)}\right\rangle=\left|\theta_{2}, \phi_{2}\right\rangle$, which, apart from a normalization factor, is given by $\left.\left\langle\psi^{(1)} \mid \Psi\right\rangle\right\rangle$. The twophoton polarization state is then represented by the two points on the Poincare sphere that correspond to the states $\left|\psi^{(1)}\right\rangle$ and $\left|\psi^{(2)}\right\rangle$.

As an example we discuss the singlet and triplet Bell states, which are given by

$$
\begin{aligned}
& \left.\left|\Psi_{S}\right\rangle\right\rangle=\frac{1}{\sqrt{2}}(|x\rangle|y\rangle-|y\rangle|x\rangle)=\frac{1}{i \sqrt{2}}(|+\rangle|-\rangle-|-\rangle|+\rangle), \\
& \left.\left|\Psi_{T}\right\rangle\right\rangle=\frac{1}{\sqrt{2}}(|x\rangle|y\rangle+|y\rangle|x\rangle)=\frac{1}{\sqrt{2}}(|-\rangle|-\rangle-|+\rangle|+\rangle),
\end{aligned}
$$

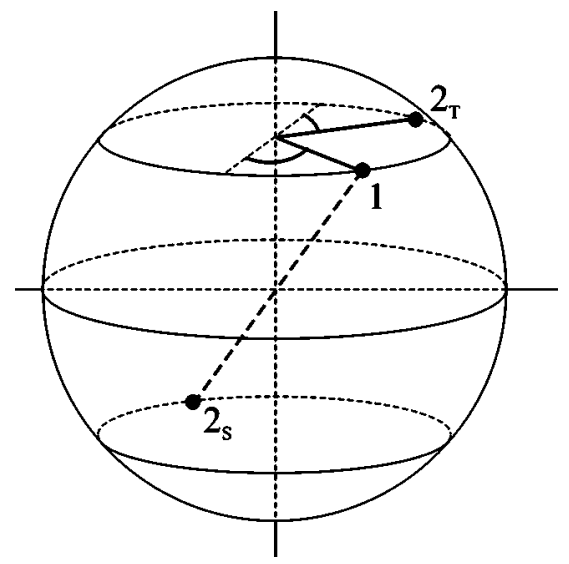

FIG. 2. Representation of the singlet and triplet Bell states on the Poincare sphere. The first photon is detected in the state with label 1. Then the second photon is projected in the state labeled with $2_{S}$ and $2_{T}$ for the singlet and triplet Bell states, respectively.

where $|x\rangle$ and $|y\rangle$ form the basis of linear polarization. In Fig. 2, the singlet and triplet Bell states are represented on the Poincaré sphere. For the singlet state (3), if we detect one photon in a specific polarization state, the other photon is projected in a state that is orthogonal to it. As a consequence, the detected and projected states are antipodes on the Poincaré sphere, that is

$$
\begin{gathered}
\theta_{1}+\theta_{2}=\pi \\
\phi_{1}-\phi_{2}=\pi \bmod (2 \pi) .
\end{gathered}
$$

For the triplet state (4), we find

$$
\begin{gathered}
\theta_{1}-\theta_{2}=0 \\
\phi_{1}+\phi_{2}=\pi \bmod (2 \pi),
\end{gathered}
$$

and we see that the detected and projected states have the same latitude. Note that these relations are invariant if we interchange the detected and projected states.

The states $\left|\psi^{(1)}\right\rangle$ and $\left|\psi^{(2)}\right\rangle$ cannot be interchanged in general. For a state $|\Psi\rangle\rangle$ of the combined system, interchangeability of the detected and projected states holds, if, for each pair of states $\left|\psi^{(1)}\right\rangle$ and $\left|\psi^{(2)}\right\rangle$ that are related by $\left.\left|\psi^{(2)}\right\rangle \propto\left\langle\psi^{(1)} \mid \Psi\right\rangle\right\rangle$, the opposite relation $\left.\left|\psi^{(1)}\right\rangle \propto\left\langle\psi^{(2)} \mid \Psi\right\rangle\right\rangle$ is also satisfied. In the Appendix we prove that these conditions do not hold unless the state $|\Psi\rangle\rangle$ is maximally entangled. The singlet and triplet Bell states discussed above are maximally entangled states, and thus satisfy interchangeability. In general, the two-photon polarization state is not maximally entangled, thus the order of detected and projected states is important.

\section{Intrinsic angular momentum associated with polarization}

As basis vectors for polarization we have taken the circular polarization vectors of Eq. (1). Then the expectation value of the intrinsic angular momentum is in the $z$ direction. For the total intrinsic angular momentum operator in the propagation direction $\Sigma_{3}$, we have 


$$
\Sigma_{3}=\sum_{i} \sigma_{3}^{i}
$$

where the summation runs over the photons and where $\sigma_{3}^{i}$ is the third Pauli matrix for photon $i$. For the third Pauli matrix we can write $\sigma_{3}=|+\rangle\langle+|-|-\rangle\langle-|$.

The expectation value of the intrinsic angular momentum, in units of $\hbar$, for the one-photon polarization state (2) is given by $\cos \theta$, where $\theta$ is the polar angle on the Poincare sphere. The expectation value for both the singlet and triplet Bell states vanishes. After detection of one photon in the state $\left|\psi^{(1)}\right\rangle=\left|\theta_{1}, \phi_{1}\right\rangle$ the two-photon polarization state $\left.|\Psi\rangle\right\rangle$ collapses to the product state $\left|\psi^{(1)}\right\rangle\left|\psi^{(2)}\right\rangle$, with $\left|\psi^{(2)}\right\rangle$ $\left.\propto\left\langle\psi^{(1)} \mid \Psi\right\rangle\right\rangle$. For the singlet Bell state, the detected and projected states are on opposite sides of the Poincare sphere, so the expectation value of the intrinsic angular momentum after the detection vanishes as well. On the other hand, for the triplet Bell state the detected and projected states have the same latitude, so that after detection the expectation value of the intrinsic angular momentum is $2 \cos \theta_{1}$. We see that the intrinsic angular momentum is not conserved. We will come back to this point in Sec. IV C.

\section{SYMMETRY PROPERTIES OF THE SUSCEPTIBILITY TENSOR}

\section{A. Invariances of the susceptibility}

The most common way to create a two-photon polarization state is by the process of SPDC. The basic process of SPDC is the annihilation of one pump photon and the creation of two photons into the signal and the idler mode. The interaction Hamiltonian arises from the nonlinear polarization of the medium coupled to the pump field, and corresponds to three-wave mixing. The polarization dependence of the interaction Hamiltonian is described by

$$
H_{I}=\int d \vec{r} \chi^{(2)}: \vec{E} \vec{E}^{\dagger} \vec{E}^{\dagger}+\text { H.c., }
$$

where $\vec{E}$ is the positive-frequency part of the electric-field operator, and the integration extends over the volume of the medium. The three dots symbolize an inner product of the second-order susceptibility tensor $\chi^{(2)}$ with the electric-field vectors. The first part of the Hamiltonian describes downconversion, its Hermitian conjugate describes up-conversion. The Hamiltonian can be written in the elegant form in Eq. (5) because we consider operation in the optical regime where $\chi^{(2)}$ is virtually frequency independent [11].

The tensor $\chi^{(2)}$ has rank 3, and therefore it has 27 components. For all materials with some spatial symmetry, not all of these components are independent and nonzero. The material transforms onto itself under the application of a covering operation of its symmetry group. Since the tensor $\chi^{(2)}$ is a property of the crystal material, it must be invariant under any one of these covering operations. This is known as Von Neumann's principle [11]. These operations form the symmetry group of the crystal, and each covering operation $R$ is represented by a Cartesian matrix $\mathbf{O}(R)$. The suscepti- bility tensor must be identical to its transformation for each covering operation $R$. This gives

$$
\chi_{i j k}^{(2)}=\sum_{l m n} \chi_{l m n}^{(2)} O(R)_{l i} O(R)_{m j} O(R)_{n k}
$$

All indicated indices attain the values $x, y, z$. In this sense, the symmetry properties of the susceptibility reflect the symmetry of the crystal. The identities in Eq. (6) introduce relations between the different components of $\chi^{(2)}$, thereby reducing the number of independent components.

The number of independent components of $\chi^{(2)}$ can be found by applying group theory [12-14]. The independent components themselves and their relations with the other components can be obtained by using the method of direct inspection $[11,15,16]$. The latter method can be illustrated by considering materials that are invariant under space inversion. The matrix elements of the transformation matrix of the inversion operation $I$ are simply $O(I)_{i j}=-\delta_{i j}$. Hence, we find from Eq. (6) that $\chi_{i j k}^{(2)}=(-1)^{3} \chi_{i j k}^{(2)}$. This shows that $\chi^{(2)}$ vanishes for a material with inversion symmetry. Only the susceptibilities of an even rank (i.e., $\chi^{(1)}, \chi^{(3)}, \ldots$ ) can be nonzero in a medium with inversion symmetry.

\section{B. Rotations and spherical basis}

Most crystalline materials that are of relevance for SPDC are invariant under a rotation over an angle $2 \pi / N$ about their symmetry axis, which we take to be the $z$ axis. To describe the effect of a rotation, it is efficient to replace the basis of Cartesian unit vectors $\vec{x}, \vec{y}$, and $\vec{z}$ by the basis of spherical unit vectors

$$
\begin{gathered}
\vec{u}_{+1}=-\frac{1}{\sqrt{2}}(\vec{x}+i \vec{y}), \\
\vec{u}_{-1}=\frac{1}{\sqrt{2}}(\vec{x}-i \vec{y}), \\
\vec{u}_{0}=\vec{z} .
\end{gathered}
$$

Note that we encountered $\vec{u}_{ \pm 1}$ before in Eq. (1) as the basis vectors for circular polarization. These unit vectors transform under rotations in the same way as the spherical harmonics $Y_{l m}$ with $l=1$. In particular, they are eigenvectors of the rotation matrix about the symmetry axis.

When $\mathbf{O}(\phi)$ denotes the rotation matrix for a counterclockwise rotation about the $z$ axis over an angle $\phi$, the transformation reads

$$
\mathbf{O}(\phi) \cdot \vec{u}_{\sigma}=\exp (-i \phi \sigma) \vec{u}_{\sigma}, \quad \sigma=-1,0,+1
$$

This eigenvalue character makes the spherical unit vectors into the natural basis for analyzing the components of the susceptibility tensor. In particular, on this basis, it is simple to identify the components that must vanish. The spherical components of the susceptibility are defined by the relations 


$$
\chi_{\rho \sigma \tau}^{(2)}=\chi^{(2)}: \vec{u}_{\rho} \vec{u}_{\sigma} \vec{u}_{\tau}, \quad \rho, \sigma, \tau=-1,0,+1
$$

\section{Nonvanishing spherical components of $\chi^{(2)}$}

For a material with an $N$-fold rotation axis, we apply a counterclockwise rotation about this axis over an angle $2 \pi / N$. We shall now determine the number of nonzero and independent components of $\chi^{(2)}$ for all values of $N$. With the invariance requirement (6), Eq. (7) leads to the simple identity

$\chi_{\rho \sigma \tau}^{(2)}=\exp [-(\rho+\sigma+\tau) 2 \pi i / N] \chi_{\rho \sigma \tau}^{(2)}, \quad \rho, \sigma, \tau=-1,0,+1$,

so that the component $\chi_{\rho \sigma \tau}^{(2)}$ must vanish whenever the exponential factor differs from 1 . It follows that the component $\chi_{\rho \sigma \tau}^{(2)}$ can only be nonzero under the condition that

$$
\rho+\sigma+\tau=k N
$$

with $k$ being an integer. Obviously, the sum $\rho+\sigma+\tau$ can acquire the values $0, \pm 1, \pm 2, \pm 3$ for the possible values of $\rho$, $\sigma$, and $\tau$.

(1) For each value of $N$, the condition (9) is satisfied when the sum of the indices is zero. This is true when all indices are zero, or when they are one of the six permutations of the three different values $-1,0,+1$. The corresponding seven components can be nonzero for any value of $N$, and also when the system possesses full axial symmetry.

(2) For $N=4$ and higher, the condition (9) cannot be obeyed for any value of $k$ other than 0 , so that all components other than these seven must vanish.

(3) For $N=3$, the two components $\chi_{+1+1+1}^{(2)}$ and $\chi_{-1-1-1}^{(2)}$ correspond to the condition (9) with $k= \pm 1$; they can be nonzero in addition to the seven components mentioned above.

(4) For $N=2$, the condition (9) with $k= \pm 1$ is obeyed by the six components with $\rho+\sigma+\tau= \pm 2$; here $\rho, \sigma, \tau$ are permutations of $+1,+1,0$ or of $-1,-1,0$. Only these six components can be nonzero for $N=2$, in addition to the seven components mentioned above.

(5) Finally, there are 12 components for which $\rho+\sigma+\tau$ $= \pm 1$. These are the ones where $\rho, \sigma, \tau$ are permutations of $0,0, \pm 1$ or of $\mp 1, \pm 1 \pm 1$. These components can only be nonzero in the trivial case that $N=1$. In this case without any symmetry, no restriction is set for any one of the 27 components.

In general, materials have other symmetry operations besides an $N$-fold rotation axis. The requirement that $\chi^{(2)}$ is invariant under the additional operations introduces relations between the nonzero components found above, thereby further reducing the number of independent components.

\section{Transverse part of $\chi^{(2)}$}

In the case that pump, signal, and idler propagate parallel to each other and to the symmetry axis, the polarization vectors lie in the $x-y$ plane. This we call the transverse configuration. The only relevant part of the susceptibility in this case is the transverse susceptibility $\chi_{T}^{(2)}$, defined as the projection of $\chi^{(2)}$ on this plane. Hence, $\chi_{T}^{(2)}$ contains the eight spherical components $\chi_{\rho \sigma \tau}^{(2)}$ with $\rho, \sigma, \tau=-1,+1$ only. For the components of $\chi_{T}^{(2)}$, the sum $\rho+\sigma+\tau$ can only attain the values $\pm 1, \pm 3$.

For $N=2$ and $N=4$ and higher, it follows from Eq. (9) that all components of $\chi_{T}^{(2)}$ vanish. Hence a nonvanishing transverse susceptibility $\chi_{T}^{(2)}$ only occurs for materials without any symmetry, or for materials with a threefold rotation axis. In the following section, we discuss the polarization properties of twin photons created by SPDC in a crystal with threefold rotational symmetry.

\section{SPDC IN A CRYSTAL WITH $C_{3 v}$ SYMMETRY}

\section{A. Hamiltonian}

We consider a crystal with $C_{3 v}$ symmetry in the transverse configuration. It has six covering operations, which are generated by a rotation over $2 \pi / 3$ and a reflection in a vertical plane that contains the $z$ axis, for which we take the $x-z$ plane. The corresponding matrix $\mathbf{O}\left(R_{v}\right)$ acting on the spherical unit vectors is represented by the transformation

$$
\mathbf{O}\left(R_{v}\right) \cdot \vec{u}_{+1}=-\vec{u}_{-1}, \quad \mathbf{O}\left(R_{v}\right) \cdot \vec{u}_{-1}=-\vec{u}_{+1} .
$$

For the transverse configuration we are only interested in $\chi_{T}^{(2)}$. As shown in Sec. III C, it follows from the threefold rotation symmetry that $\chi_{+1+1+1}^{(2)}$ and $\chi_{-1-1-1}^{(2)}$ are the only nonzero components of $\chi_{T}^{(2)}$. According to Eq. (10), the invariance relation (6) applied to reflection about the $x-z$ plane yields the relation

$$
\chi_{+1+1+1}^{(2)}=-\chi_{-1-1-1}^{(2)} \equiv G .
$$

Hence, for $C_{3 v}$, we find that $\chi_{T}^{(2)}$ is determined by a single independent parameter $G$. This is in agreement with the result obtained using the procedure by Bhagavantam and Suryanarayana [12].

Now we obtain the Hamiltonian in the transverse configuration for a crystal with $C_{3 v}$ symmetry. For the positivefrequency part of the electric-field operator, we write

$$
\vec{E}(\vec{r}, t) \propto \int d \vec{k} \sum_{\lambda} \sqrt{\omega_{\lambda}(\vec{k})} a_{\lambda}(\vec{k}) \vec{\varepsilon}_{\lambda}(\vec{k}) \exp \left[i \vec{k} \cdot \vec{r}-i \omega_{\lambda}(\vec{k}) t\right],
$$

where $a_{\lambda}(\vec{k})$ annihilates a photon with wave vector $\vec{k}$ and polarization vector $\vec{\varepsilon}_{\lambda}(\vec{k})$ and where the summation over $\lambda$ runs over the two basis states of polarization. In the transverse configuration, the index of refraction of the crystal does not depend on the polarization for the common direction of propagation. We then have $\vec{\varepsilon}_{\lambda}(\vec{k}) \rightarrow \vec{\varepsilon}_{\lambda}$ and $\omega_{\lambda}(\vec{k})$ $\rightarrow \omega(\vec{k})$, and we find that

$$
\vec{E}(\vec{r}, t) \propto \int d \vec{k} \sqrt{\omega(\vec{k})} \exp [i \vec{k} \cdot \vec{r}-i \omega(\vec{k}) t] \sum_{\lambda} a_{\lambda}(\vec{k}) \vec{\varepsilon}_{\lambda} .
$$

We see that the electric-field operator is split into a polarization part and a part concerning the modes in $k$ space. As a 
basis for polarization we use the circular polarization states $| \pm\rangle$. Since we are only interested in the polarization part, we write

$$
\vec{E} \propto a_{+} \vec{u}_{+1}+a_{-} \vec{u}_{-1}
$$

Here $a_{+}$and $a_{-}$are the annihilation operators for a photon with right- and left-handed circular polarizations, respectively.

From Eq. (11) and the definition in Eq. (8), it follows that the transverse susceptibility can be expressed in terms of the spherical unit vectors $\vec{u}_{ \pm 1}$ as

$$
\chi_{T}^{(2)}=G\left(\vec{u}_{+1}^{*} \vec{u}_{+1}^{*} \vec{u}_{+1}^{*}-\vec{u}_{-1}^{*} \vec{u}_{-1}^{*} \vec{u}_{-1}^{*}\right) .
$$

Using the fact that the spherical basis is unitary and that

$$
\left(\vec{u}_{+1}\right)^{*}=-\vec{u}_{-1},
$$

we find that

$$
\begin{gathered}
\vec{u}_{+1} \cdot \vec{u}_{+1}=\vec{u}_{-1} \cdot \vec{u}_{-1}=0, \\
\vec{u}_{+1} \cdot \vec{u}_{-1}=-1 .
\end{gathered}
$$

We substitute the expressions that we obtained above in Eq. (5) and, apart from an irrelevant overall factor, the Hamiltonian in the transverse configuration is found as

$$
H_{I} \propto G\left(a_{p+} a_{s-}^{\dagger} a_{i-}^{\dagger}-a_{p-} a_{s+}^{\dagger} a_{i+}^{\dagger}\right)+\text { H.c. },
$$

where the labels $p, s$, and $i$ refer to the modes in $k$ space of the pump, signal, and idler photon, respectively. This shows that the absorption of a left circularly polarized pump photon is accompanied by the creation of a signal and an idler photon, which are both right circularly polarized, and vice versa.

\section{B. Representation on the Poincaré sphere}

We now use the Poincare representation for the description of the entangled state of the two SPDC photons for a given polarization of the pump. We consider a pump photon in the state $\left|\theta_{p}, \phi_{p}\right\rangle$. The interaction Hamiltonian (13) in the transverse configuration applied to this initial state yields, to first order, the two-photon state

$$
\begin{aligned}
|\Psi\rangle\rangle= & \cos \left(\theta_{p} / 2\right) \exp \left(-i \phi_{p} / 2\right)|-\rangle|-\rangle-\sin \left(\theta_{p} / 2\right) \\
& \times \exp \left(+i \phi_{p} / 2\right)|+\rangle|+\rangle .
\end{aligned}
$$

This expression gives the two-photon state as a linear superposition of two-photon states that are pairwise orthogonal, so that this state is already in Schmidt-decomposed form. According to the definition by Abouraddy et al. [17] the degree of entanglement is $\sin \theta_{p}$. For linear polarization $\left(\theta_{p}\right.$ $=\pi / 2$ ), the created two-photon state is maximally entangled. We find the triplet Bell state (4) in the case that the direction of the linear polarization of the pump is parallel to the vertical reflection plane containing the $x$ axis, so that $\phi_{p}=0$. The singlet Bell state (3) cannot be obtained by choosing an appropriate pump polarization since for the state $|\Psi\rangle\rangle$ of Eq.

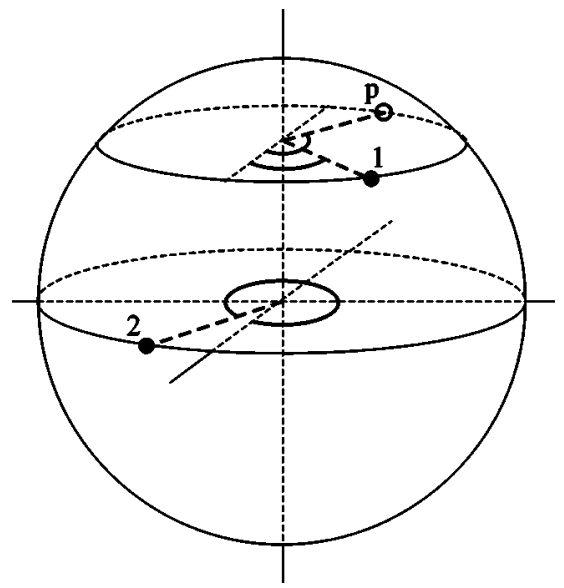

FIG. 3. Representation of a two-photon state created by SPDC where the projected polarization state 2 is found upon detection in state 1 . The polarization of the pump is represented by the open dot and labeled with $p$.

(14), the overlap $\left\langle\left\langle\Psi_{S} \mid \Psi\right\rangle\right\rangle$ with the singlet Bell state vanishes for all pump polarizations.

When one photon is detected in the selected polarization state $\left|\psi^{(1)}\right\rangle=\left|\theta_{1}, \phi_{1}\right\rangle$, the state of the remaining photon collapses into the state $\left.\left|\psi^{(2)}\right\rangle=\left|\theta_{2}, \phi_{2}\right\rangle \propto\left\langle\psi^{(1)} \mid \Psi\right\rangle\right\rangle$. The spherical angles $\theta_{2}$ and $\phi_{2}$ of $\left|\psi^{(2)}\right\rangle$ are found to be given by the equalities

$$
\frac{\tan \left(\theta_{1} / 2\right)}{\tan \left(\theta_{2} / 2\right)}=\tan \left(\theta_{p} / 2\right), \quad \phi_{1}+\phi_{2}+\phi_{p}=\pi \bmod (2 \pi) \text {. }
$$

Note that the states $\left|\psi^{(1)}\right\rangle$ and $\left|\psi^{(2)}\right\rangle$ cannot be interchanged, since in general the polarization state of the two created photons is not maximally entangled, except for the case of a linearly polarized pump. The relation between the two polarization states $\left|\psi^{(1)}\right\rangle$ and $\left|\psi^{(2)}\right\rangle$ is illustrated in Figs. 3 and 4.

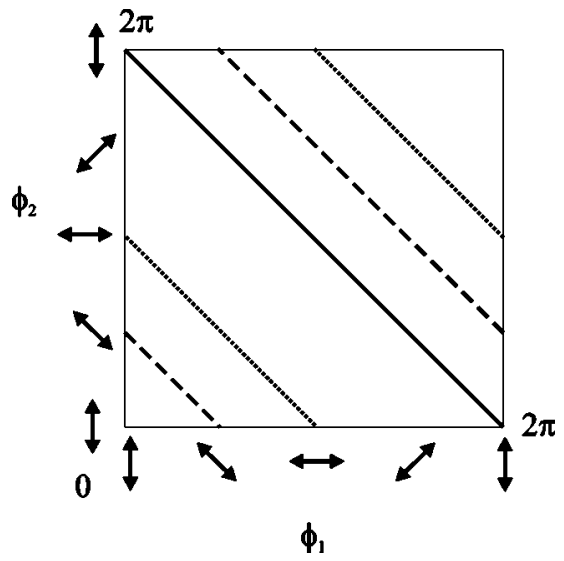

FIG. 4. Representation of the two-photon state created by SPDC with a linearly polarized pump. Then the polarization states 1 and 2 have the same latitude on the Poincare sphere. For $\phi_{p}=0$, the relation between $\phi_{1}$ and $\phi_{2}$ is represented by the dotted lines, for $\phi_{p}=\pi / 2$ by the broken lines, and for $\phi_{p}=\pi$ by the continuous line. The double arrow below and next to the axes represents the orientation of the long axis of the polarization ellipse. 
The relation between the azimuthal angles in Eq. (15) is invariant when all angles are increased by an amount of $2 \pi / 3$. This corresponds to a rotation in real space about the $z$ axis, or symmetry axis, over half this angle, that is, over $\pi / 3$, as can be seen from Eqs. (7) and (2). This is somewhat surprising, since the crystal is invariant only under a rotation over $2 \pi / 3$. To check consistency, we perform a rotation of the crystal over an angle $\beta$ about the $z$ axis. Using the transformation property under rotations in Eq. (7), we find that the relation between $\chi_{+1+1+1}^{(2)}$ and $\chi_{-1-1-1}^{(2)}$ in Eq. (11) changes to

$$
\exp (-3 i \beta) \chi_{+1+1+1}^{(2)}=-\exp (+3 i \beta) \chi_{-1-1-1}^{(2)} .
$$

Hence, a rotation over $\pi / 3$ produces a sign change for $\chi_{T}^{(2)}$ as a whole, which does not change the polarization properties of signal and idler. The same conclusion follows by noting that a polarization state is basically unchanged by a rotation over $\pm \pi$. Therefore, a rotation of all polarization vectors over $\pi / 3$ is equivalent to a rotation of the polarization states over $-2 \pi / 3$, or, for that matter, a rotation of the crystal over $2 \pi / 3$.

The Hermitian conjugate of the interaction Hamiltonian in Eq. (13) represents the nonlinear process of up-conversion. This process transforms the two-photon polarization state $\left|\psi^{(1)}\right\rangle\left|\psi^{(2)}\right\rangle$ into the one-photon state $\left|\theta_{p}, \phi_{p}\right\rangle$. The spherical angles $\theta_{p}$ and $\phi_{p}$ can then again be obtained from Eq. (15), but with the labels 1 and $p$ interchanged.

\section{Intrinsic angular momentum}

For a pump photon in the state $\left|\theta_{p}, \phi_{p}\right\rangle$, the expectation value of the intrinsic angular momentum, in units of $\hbar$, is given by $\cos \theta_{p}$, while for the two-photon state (14) created by SPDC in the crystal we find $-2 \cos \theta_{p}$. We see that the intrinsic angular momentum is not conserved in the process of SPDC, while, in the transverse configuration, the orbital angular momentum is conserved [3-5]. In order to satisfy conservation of total angular momentum, we conclude that there must be a transfer of angular momentum to the crystal [10]. The expectation value of the amount of transferred angular momentum to the crystal is then $3 \cos \theta_{p}$. Like we saw in Sec. II C when discussing the triplet Bell state (4), the actual amount of transfer of angular momentum to the crystal will depend on the detected polarization state $\left|\psi^{(1)}\right\rangle$, which shows the highly nonlocal nature of the transfer.

Conservation or nonconservation of angular momentum in SPDC depends on the transformation properties of the Hamiltonian under rotation. The orbital angular momentum depends on the position dependence of the complex field amplitude, and its conservation results from the fact that the Hamiltonian does not depend on position. On the other hand, the intrinsic angular momentum depends on the polarization properties of the fields, which are determined by the tensor character of $\chi^{(2)}$. The effect of a rotation about the symmetry axis in the spherical basis is a complex phase change, since the spherical basis vectors are eigenvectors of rotation. For $C_{3 v}$ symmetry, the relevant elements $\chi_{+1+1+1}^{(2)}$ and $\chi_{-1-1-1}^{(2)}$ are only invariant under a rotation over an angle of $2 \pi / 3$ and not under a rotation over an arbitrary angle. As a consequence, in case of a crystal with $C_{3 v}$ symmetry, the intrinsic angular momentum is not conserved.

\section{CONCLUSIONS}

We have discussed the use of a spherical basis for describing polarization entanglement of twin photons as produced in the process of spontaneous parametric down-conversion. This choice leads to a very transparent discussion regarding the conservation of intrinsic angular momentum in SPDC. We have used the Poincare sphere to describe arbitrary polarization states of pump, signal, and idler. On this sphere, the singlet Bell state corresponds to a pair of antipodes, while in the triplet Bell state, the photons have equal latitude.

We have employed the spherical basis to analyze the polarization entanglement of signal and idler as it arises in the process of SPDC in a crystal of $C_{3 v}$ symmetry when all optical beams are collinear with the symmetry axis of the crystal. We have shown that the threefold crystalline symmetry is a prerequisite for SPDC in this geometry. For this crystal and geometry, we have derived simple relationships between the spherical coordinates of pump, signal, and idler photons on the Poincare sphere; these relationships provide direct insight into the issue of conservation of intrinsic angular momentum.

Twin photon generation in the chosen configuration cannot be phase matched; experimental realization of SPDC in the proposed geometry will therefore be nontrivial. With a beta-barium-borate (BBO) crystal $\left(C_{3 v}\right.$ symmetry), cut for $0^{\circ}$ phase matching, having a length equal to the coherence length $(\approx 13 \mu \mathrm{m}$ at $\lambda=800 \mathrm{~nm}$ [18]), such an experiment should be feasible.

\section{ACKNOWLEDGMENTS}

This work was supported by the "Stichting voor Fundamenteel Onderzoek der Materie" (FOM), and the European Union under the IST-ATESIT contract.

\section{APPENDIX: INTERCHANGEABILITY OF DETECTED AND PROJECTED STATES}

When a quantum system consisting of two subsystems 1 and 2 with the same dimension $d$ is in a state $|\Psi\rangle\rangle$, and system 1 is detected in the state $\left|\psi^{(1)}\right\rangle$, system 2 is projected into the state $\left|\psi^{(2)}\right\rangle$ that is proportional to the partial inner product $\left.\left\langle\psi^{(1)} \mid \Psi\right\rangle\right\rangle$. In general, the inverse statement is not true: detection of system 2 in $\left|\psi^{(2)}\right\rangle$ projects system 1 in a state that is not necessarily equal to $\left|\psi^{(1)}\right\rangle$. In this appendix we prove that the roles of $\left|\psi^{(1)}\right\rangle$ and $\left|\psi^{(2)}\right\rangle$ can be interchanged for all choices of the detected state if and only if the state $|\Psi\rangle\rangle$ is maximally entangled.

Consider a state $|\Psi\rangle\rangle$ for which the detected state and the resulting projected state can be interchanged. For any normalized detected state $\left|\psi^{(1)}\right\rangle$ of system 1 , the resulting normalized projected state $\left|\psi^{(2)}\right\rangle$ of system 2 obeys the identity

$$
\left.\left\langle\psi^{(1)} \mid \Psi\right\rangle\right\rangle=c\left|\psi^{(2)}\right\rangle,
$$


and the normalization constant is obviously $c$ $=\left\langle\psi^{(1)} \mid\left\langle\psi^{(2)} \mid \Psi\right\rangle\right\rangle$. Because of the assumption of interchangeability, we can also write

$$
\left.\left\langle\psi^{(2)} \mid \Psi\right\rangle\right\rangle=c\left|\psi^{(1)}\right\rangle,
$$

with the same normalization constant.

We choose an orthonormal basis $\left|\psi_{n}^{(1)}\right\rangle$ of system 1 . Detection of system 1 in the state $\left|\psi_{n}^{(1)}\right\rangle$ projects system 2 in the state $\left|\psi_{n}^{(2)}\right\rangle$, defined by

$$
\left.\left\langle\psi_{n}^{(1)} \mid \Psi\right\rangle\right\rangle=c_{n}\left|\psi_{n}^{(2)}\right\rangle .
$$

This allows us to express the state $|\Psi\rangle\rangle$ in the form

$$
|\Psi\rangle\rangle=\sum_{n=1}^{d} c_{n}\left|\psi_{n}^{(1)}\right\rangle\left|\psi_{n}^{(2)}\right\rangle .
$$

Now we use the interchangeability of the detected and projected states, which gives

$$
\left.\left\langle\psi_{n}^{(2)} \mid \Psi\right\rangle\right\rangle=c_{n}\left|\psi_{n}^{(1)}\right\rangle .
$$

Substituting Eq. (A2) into Eq. (A3) shows that the states $\left|\psi_{n}^{(2)}\right\rangle$ form an orthonormal basis of system 2 .

Finally, we apply the assumption of interchangeability for an arbitrary state $\left|\psi^{(1)}\right\rangle=\sum_{n=1}^{d} a_{n}\left|\psi_{n}^{(1)}\right\rangle$. This gives for the projected state of system 2 ,

$$
\left.c\left|\psi^{(2)}\right\rangle=\sum_{n=1}^{d} a_{n}^{*}\left\langle\psi_{n}^{(1)} \mid \Psi\right\rangle\right\rangle=\sum_{n=1}^{d} a_{n}^{*} c_{n}\left|\psi_{n}^{(2)}\right\rangle,
$$

where we used Eq. (A1) in the last step. Conversely, when we first detect system 2 in the state $\left|\psi^{(2)}\right\rangle$, system 1 is projected into a state proportional to

$$
\left.\left.\left\langle\psi^{(2)} \mid \Psi\right\rangle\right\rangle=\frac{1}{c^{*}} \sum_{n=1}^{d} a_{n} c_{n}^{*}\left\langle\psi_{n}^{(2)} \mid \Psi\right\rangle\right\rangle=\frac{1}{c^{*}} \sum_{n=1}^{d} a_{n}\left|c_{n}\right|^{2}\left|\psi_{n}^{(1)}\right\rangle .
$$

This is proportional to the original state $\left|\psi_{n}^{(1)}\right\rangle$ only when all coefficients $\left|c_{n}\right|^{2}$ are identical. Then Eq. (A2) can be expressed as a biorthogonal expansion

$$
|\Psi\rangle\rangle=\frac{1}{\sqrt{d}} \sum_{n=1}^{d} \exp \left(i \phi_{n}\right)\left|\psi_{n}^{(1)}\right\rangle\left|\psi_{n}^{(2)}\right\rangle
$$

in which each term has the same strength. This is a state with maximal entanglement.

On the other hand, when the state of the combined system can be expressed in the form (A4), one easily checks that it satisfies interchangeability.
[1] H.H. Arnaut and G.A. Barbosa, Phys. Rev. Lett. 85, 286 (2000).

[2] G.A. Barbosa and H.H. Arnaut, Phys. Rev. A 65, 053801 (2002).

[3] S. Franke-Arnold, S.M. Barnett, M.J. Padgett, and L. Allen, Phys. Rev. A 65, 033823 (2002).

[4] A. Mair, A. Vaziri, G. Weihs, and A. Zeilinger, Nature (London) 412, 313 (2001).

[5] E.R. Eliel, S.M. Dutra, G. Nienhuis, and J.P. Woerdman, Phys. Rev. Lett. 86, 5208 (2001).

[6] J. Leach, M.J. Padgett, S.M. Barnett, S. Franke-Arnold, and J. Courtial, Phys. Rev. Lett. 88, 257901 (2002).

[7] A.T. O'Neil, I. MacVicar, L. Allen, and M.J. Padgett, Phys. Rev. Lett. 88, 053601 (2002).

[8] G. Molina-Terriza, J.P. Torres, and L. Torner, Phys. Rev. Lett. 88, 013601 (2002).

[9] L. Allen, J. Opt. B: Quantum Semiclassical Opt. 4, S1 (2002).
[10] N. Bloembergen, J. Opt. Soc. Am. 70, 1429 (1980).

[11] P.N. Butcher and D. Cotter, The Elements of Non-Linear Optics (Cambridge University Press, Cambridge, UK, 1990).

[12] S. Bhagavantam and D. Suryanarayana, Acta Crystallogr. 2, 21 (1949).

[13] J.S. Lomont, Applications of Finite Groups (Academic Press, New York, 1959).

[14] P. Erdös, Helv. Phys. Acta 37, 493 (1964).

[15] F.G. Fumi, Acta Crystallogr. 5, 44 (1952).

[16] S.V. Popov, Y.P. Svirko, and N.I. Zheludev, Susceptibility Tensors for Non-Linear Optics (Institute of Physics, Bristol, UK, 1995).

[17] A.F. Abouraddy, B.E.A. Saleh, A.V. Sergienko, and M.C. Teich, Phys. Rev. A 64, 050101 (2001).

[18] V.G. Dmitriev, G.G. Gurzadyan, and D.N. Nikogosyan, Handbook of Nonlinear Optical Crystals, 3rd ed. (Springer-Verlag, Berlin, 1999). 